\title{
Regioselective Reduction of 3-Methoxymaleimides: an Efficient Method for the Synthesis of Methyl 5-Hydroxytetramates
}

\author{
Fatiah Issa, Joshua Fischer, Peter Turner and Mark J. Coster* \\ School of Chemistry, The University of Sydney, NSW 2006, Australia
}

\section{SUPPORTING INFORMATION}

Table of Contents

Page

General Experimental

S1-S2

Experimental Procedures and Characterization Data

S2-S5

Single crystal x-ray diffraction experimental for compound $\mathbf{6 e}$

Figure S1: ORTEP Depiction of $\mathbf{6 e}$

References

${ }^{1} \mathrm{H}$ and ${ }^{13} \mathrm{C}$ NMR Spectra

S9-S22

\section{General Experimental}

Solvents and reagents were dried and purified according to literature methods, prior to use, and reactions were conducted under an inert atmosphere of dry nitrogen, unless otherwise stated. Thin layer chromatography was performed using $0.2 \mathrm{~mm}$ thick pre-coated aluminium-backed silica gel plates. Compounds were visualised by ultraviolet absorption or by staining with vanillin in ethanolic sulfuric acid or potassium permanganate. Flash chromatography was performed using 40-63 $\mu \mathrm{m}$ silica gel with the specified solvents. Preparative HPLC was performed using a $10 \mu$, $21.5 \times 25 \mathrm{~cm}$ column with UV detection at $254 \mathrm{~nm}$.

Only strong absorptions are reported for IR spectra and absorption maxima are expressed in wavenumbers $\left(\mathrm{cm}^{-1}\right)$. Compounds were prepared as thin films between sodium chloride plates or as $\mathrm{KBr}$ discs. NMR spectra were recorded at $300 \mathrm{~K}$ in deuteriochloroform, unless otherwise stated. Data are expressed in parts per million downfield shift from tetramethylsilane and are reported as chemical shift, multiplicity $(\mathrm{s}=$ singlet, $\mathrm{d}=$ doublet, $\mathrm{t}=$ triplet, $\mathrm{m}=$ multiplet, $\mathrm{br}=$ broad and app = apparent $)$, coupling constant $(\mathrm{J} \mathrm{Hz})$ and relative integral. Low resolution mass spectra were recorded using positive electrospray ionization (ESI+). High resolution mass spectra 
were recorded using electron impact (EI+) at $70 \mathrm{eV}$ in magnetic scan with PFK as standard or ESI+. Melting points were determined in open glass capillaries and are uncorrected.

\section{Experimental Procedures and Characterization Data}

3-Methoxy-1-methyl-1H-pyrrole-2,5-dione (5b). Method A in acetone, using methyl iodide, provided 5b as a colourless solid (87\%): mp 126-127 ${ }^{\circ} \mathrm{C}$, lit. ${ }^{1} \mathrm{mp} 129-130{ }^{\circ} \mathrm{C}$; IR (thin film) 3113, 1705, $1634 \mathrm{~cm}^{-1}$; ${ }^{1} \mathrm{H}$ NMR (300 MHz, $\left.\mathrm{CDCl}_{3}\right) \delta 5.41(\mathrm{~s}, 1 \mathrm{H}), 3.93(\mathrm{~s}, 3 \mathrm{H}), 3.00(\mathrm{~s}, 3 \mathrm{H})$; ${ }^{13} \mathrm{C}$ NMR $\left(75.5 \mathrm{MHz}, \mathrm{CDCl}_{3}\right) \delta 170.2(\mathrm{C}), 165.7(\mathrm{C}), 161.1(\mathrm{C}), 96.2(\mathrm{CH}), 58.9\left(\mathrm{CH}_{3}\right), 23.5$ $\left(\mathrm{CH}_{3}\right)$. Spectroscopic data matched that previously reported. ${ }^{1}$

1-Ethyl-3-methoxy-1H-pyrrole-2,5-dione (5c). Method A in acetone, using ethyl iodide, provided $\mathbf{5 c}$ as a colourless solid (92\%): mp 69-70 ${ }^{\circ} \mathrm{C}$; IR (thin film) $3109,1709,1636 \mathrm{~cm}^{-1} ;{ }^{1} \mathrm{H}$ NMR (300 MHz, $\left.\mathrm{CDCl}_{3}\right) \delta 5.40(\mathrm{~s}, 1 \mathrm{H}), 3.93(\mathrm{~s}, 3 \mathrm{H}), 3.56(\mathrm{q}, J=7.2 \mathrm{~Hz}, 2 \mathrm{H}), 1.18(\mathrm{t}, J=7.2$ $\mathrm{Hz}, 3 \mathrm{H}) ;{ }^{13} \mathrm{C} \mathrm{NMR}\left(75.5 \mathrm{MHz}, \mathrm{CDCl}_{3}\right) \delta 170.0(\mathrm{C}), 165.4(\mathrm{C}), 160.9(\mathrm{C}), 96.1(\mathrm{CH}), 58.8\left(\mathrm{CH}_{3}\right)$, $32.5\left(\mathrm{CH}_{2}\right), 13.9\left(\mathrm{CH}_{3}\right)$; MS (ESI) $m / z 156[\mathrm{M}+\mathrm{H}]^{+}$; HRMS (ESI) calcd for $\mathrm{C}_{7} \mathrm{H}_{10} \mathrm{NO}_{3}[\mathrm{M}+\mathrm{H}]^{+}$ 156.0655, found 156.0654 .

3-Methoxy-1-(4-methoxybenzyl)-1H-pyrrole-2,5-dione (5f). Method A in acetone, using PMBCl, provided 5f as a colourless solid (91\%): mp 104-105 ${ }^{\circ} \mathrm{C}$; IR (thin film) 3101, 3013, 1713, $1636 \mathrm{~cm}^{-1} ;{ }^{1} \mathrm{H}$ NMR $\left(300 \mathrm{MHz}, \mathrm{CDCl}_{3}\right) \delta 7.29(\mathrm{~d}, J=8.7 \mathrm{~Hz}, 2 \mathrm{H}), 6.83(\mathrm{~d}, J=8.7 \mathrm{~Hz}$, 2H), $5.39(\mathrm{~s}, 1 \mathrm{H}), 4.59$ (s, 2H), $3.90(\mathrm{~s}, 3 \mathrm{H}), 3.77(\mathrm{~s}, 3 \mathrm{H}) ;{ }^{13} \mathrm{C} \mathrm{NMR}\left(75.5 \mathrm{MHz}, \mathrm{CDCl}_{3}\right) \delta 169.8$ (C), $165.3(\mathrm{C}), 161.0(\mathrm{C}), 159.2(\mathrm{C}), 129.9(\mathrm{CH}), 128.6(\mathrm{C}), 114.0(\mathrm{CH}), 96.3(\mathrm{CH}), 58.8\left(\mathrm{CH}_{3}\right)$, $55.2\left(\mathrm{CH}_{3}\right), 40.7\left(\mathrm{CH}_{2}\right)$; MS (ESI) $m / z 270[\mathrm{M}+\mathrm{Na}]^{+}$; HRMS (ESI) calcd for $\mathrm{C}_{13} \mathrm{H}_{13} \mathrm{NO}_{4} \mathrm{Na}[\mathrm{M}$ $+\mathrm{Na}]^{+} 270.0737$, found 270.0736 .

5-Hydroxy-4-methoxy-1H-pyrrol-2(5H)-one (6a). Purification by flash chromatography $\left(\mathrm{MeOH} / \mathrm{CH}_{2} \mathrm{Cl}_{2}\right.$ 10:90) afforded $\mathbf{6 a}(87 \%)$ as a colourless solid: mp 175-178 ${ }^{\circ} \mathrm{C}$, lit. ${ }^{2} \mathrm{mp} 163{ }^{\circ} \mathrm{C}$; IR (KBr disc) 3223 (br), 3175 (br), 1645, $1626 \mathrm{~cm}^{-1} ;{ }^{1} \mathrm{H}$ NMR (300 MHz, $d_{6}$-DMSO) $\delta 7.73$ (br s, $1 \mathrm{H}), 6.18(\mathrm{~d}, J=8.5 \mathrm{~Hz}, 1 \mathrm{H}), 5.21(\mathrm{dd}, J=8.5 \mathrm{~Hz}, J=1.7 \mathrm{~Hz}, 1 \mathrm{H}), 4.97(\mathrm{~d}, J=1.4 \mathrm{~Hz}, 1 \mathrm{H})$, $3.74(\mathrm{~s}, 3 \mathrm{H}) ;{ }^{13} \mathrm{C}$ NMR $\left(75.7 \mathrm{MHz}, d_{6}\right.$-DMSO) $\delta 176.5(\mathrm{C}), 172.4(\mathrm{C}), 93.6(\mathrm{CH}), 77.8(\mathrm{CH})$, 
$58.8\left(\mathrm{CH}_{3}\right)$; MS (EI) m/z $129[\mathrm{M}]^{+}, 112[\mathrm{M}-\mathrm{OH}]^{+}, 97$ (34), 69 (100); HRMS (EI) calcd for $\mathrm{C}_{5} \mathrm{H}_{7} \mathrm{NO}_{3}[\mathrm{M}]^{+} 129.0426$, found 129.0427 . Spectroscopic data matched that previously reported. ${ }^{2}$

5-Hydroxy-4-methoxy-1-methyl-1H-pyrrol-2(5H)-one $\quad(6 \mathrm{~b}) . \quad$ Purification by flash chromatography (EtOAc/acetone 95:5) afforded $\mathbf{6 b}$ as a colourless solid (87\%): $\mathrm{mp} 130-131{ }^{\circ} \mathrm{C}$, lit. ${ }^{3} \mathrm{mp} 140-141{ }^{\circ} \mathrm{C}$; IR (thin film) 3267, 3107, 1693, $1634 \mathrm{~cm}^{-1} ;{ }^{1} \mathrm{H}$ NMR (300 MHz, $\mathrm{CDCl}_{3}$ ) $\delta$ 5.09 (br d, $J=\sim 8 \mathrm{~Hz}, 1 \mathrm{H}), 4.95(\mathrm{~s}, 1 \mathrm{H}), 4.30(\mathrm{br} \mathrm{d}, J=\sim 9 \mathrm{~Hz}, 1 \mathrm{H}), 3.83(\mathrm{~s}, 3 \mathrm{H}), 2.90(\mathrm{~s}, 3 \mathrm{H}) ;{ }^{13} \mathrm{C}$ NMR (75.5 MHz, $\left.\mathrm{CDCl}_{3}\right) \delta 173.7(\mathrm{C}), 170.8(\mathrm{C}), 93.2(\mathrm{CH}), 82.4(\mathrm{CH}), 58.4\left(\mathrm{CH}_{3}\right), 25.6\left(\mathrm{CH}_{3}\right)$; MS (ESI) $m / z 166[\mathrm{M}+\mathrm{Na}]^{+}$; HRMS (ESI) calcd for $\mathrm{C}_{6} \mathrm{H}_{9} \mathrm{NO}_{3} \mathrm{Na}[\mathrm{M}+\mathrm{Na}]^{+} 166.0475$, found 166.0475 .

1-Ethyl-5-hydroxy-4-methoxy-1H-pyrrol-2(5H)-one $\quad(6 c)$. Purification by flash chromatography (EtOAc/acetone 95:5) afforded $\mathbf{6 c}$ as a colourless solid (84\%): $\mathrm{mp} 79-83{ }^{\circ} \mathrm{C}$; IR (thin film) 3337, 1653, $1637 \mathrm{~cm}^{-1} ;{ }^{1} \mathrm{H}$ NMR $\left(200 \mathrm{MHz}, \mathrm{CDCl}_{3}\right) \delta 5.21(\mathrm{~d}, J=10.0 \mathrm{~Hz}, 1 \mathrm{H}), 4.98$ (s, 1H), 3.83 (s, 3H), 3.59 (sextet, $J=7.0 \mathrm{~Hz}, 1 \mathrm{H}$ ), 3.28 (sextet, $J=7.0 \mathrm{~Hz}, 1 \mathrm{H}$ ), 2.64 (d, $J=10.1$ $\mathrm{Hz}, 1 \mathrm{H}), 1.17(\mathrm{t}, J=7.2 \mathrm{~Hz}, 3 \mathrm{H}) ;{ }^{13} \mathrm{C}$ NMR $\left(75.5 \mathrm{MHz}, \mathrm{CDCl}_{3}\right) \delta 173.8(\mathrm{C}), 170.5(\mathrm{C}), 93.2$ $(\mathrm{CH}), 80.3(\mathrm{CH}), 58.4\left(\mathrm{CH}_{3}\right), 33.5\left(\mathrm{CH}_{2}\right), 13.6\left(\mathrm{CH}_{3}\right)$; MS (ESI) m/z $180[\mathrm{M}+\mathrm{Na}]^{+}, 158[\mathrm{M}+$ $\mathrm{H}]^{+}$; HRMS (ESI) calcd for $\mathrm{C}_{7} \mathrm{H}_{11} \mathrm{NO}_{3} \mathrm{Na}[\mathrm{M}+\mathrm{Na}]^{+}$180.0631, found 180.0629 .

1-Allyl-5-hydroxy-4-methoxy-1H-pyrrol-2(5H)-one (6d). Purification by flash chromatography (EtOAc/acetone 95:5) afforded $\mathbf{6 d}$ as a colourless solid (85\%): $\mathrm{mp} 70-71^{\circ} \mathrm{C}$; IR (thin film) 3275, 1666, $1636 \mathrm{~cm}^{-1} ;{ }^{1} \mathrm{H}$ NMR $\left(300 \mathrm{MHz}, \mathrm{CDCl}_{3}\right) \delta 5.77$ (dddd, $J=17.2,11.6,7.0,4.6 \mathrm{~Hz}, 1 \mathrm{H}$ ), 5.21 (br s, 1H), $5.16(\mathrm{dq}, J=\sim 18,1 \mathrm{~Hz}, 1 \mathrm{H}), 5.15(\mathrm{dq}, J=\sim 10,1 \mathrm{~Hz}, 1 \mathrm{H}), 5.09$ (br s, 1H), 4.96 (s, $1 \mathrm{H}), 4.23(\mathrm{ddt}, J=15.8,4.6,1.6 \mathrm{~Hz}, 1 \mathrm{H}), 3.82(\mathrm{~s}, 3 \mathrm{H}), 3.70(\mathrm{ddt}, J=15.8,7.0,1.0 \mathrm{~Hz}, 1 \mathrm{H}) ;{ }^{13} \mathrm{C}$ NMR (75.5 MHz, $\left.\mathrm{CDCl}_{3}\right) \delta 174.1(\mathrm{C}), 170.6(\mathrm{C}), 133.0(\mathrm{CH}), 117.3\left(\mathrm{CH}_{2}\right), 92.9(\mathrm{CH}), 80.1$ $(\mathrm{CH}), 58.4\left(\mathrm{CH}_{3}\right), 41.0\left(\mathrm{CH}_{2}\right)$; MS (ESI) $\mathrm{m} / z 192\left[\mathrm{M}+\mathrm{Na}^{+}, 170[\mathrm{M}+\mathrm{H}]^{+}, 152[\mathrm{M}-\mathrm{OH}]^{+}\right.$; HRMS (ESI) calcd for $\mathrm{C}_{8} \mathrm{H}_{11} \mathrm{NO}_{3} \mathrm{Na}[\mathrm{M}+\mathrm{Na}]^{+}$192.0631, found 192.0637 .

5-Hydroxy-4-methoxy-1-(4-methoxybenzyl)-1H-pyrrol-2(5H)-one (6f). Purification by flash chromatography (EtOAc/ $\mathrm{CH}_{2} \mathrm{Cl}_{2}$ 50:50) afforded $\mathbf{6 f}$ as a colourless solid (76\%): mp 167-169 ${ }^{\circ} \mathrm{C}$; IR (thin film) $3115,1643 \mathrm{~cm}^{-1} ;{ }^{1} \mathrm{H}$ NMR $\left(300 \mathrm{MHz}, \mathrm{CDCl}_{3}\right) \delta 7.21(\mathrm{~d}, J=8.6 \mathrm{~Hz}, 2 \mathrm{H}), 6.84(\mathrm{~d}, J$ 
$=8.6 \mathrm{~Hz}, 2 \mathrm{H}), 5.04(\mathrm{~d}, J=8.8 \mathrm{~Hz}, 1 \mathrm{H}), 5.00(\mathrm{~s}, 1 \mathrm{H}), 4.88(\mathrm{~d}, J=14.9 \mathrm{~Hz}, 1 \mathrm{H}), 4.12(\mathrm{~d}, J=14.9$ $\mathrm{Hz}, 1 \mathrm{H}), 3.80$ (s, 3H), 3.78 (s, 3H), $3.13($ br s, $1 \mathrm{H}) ;{ }^{13} \mathrm{C} \mathrm{NMR}\left(75.5 \mathrm{MHz}, \mathrm{CDCl}_{3}\right) \delta 173.6(\mathrm{C})$, $170.0(\mathrm{C}), 159.0(\mathrm{C}), 129.6(\mathrm{CH}), 129.4(\mathrm{C}), 114.1(\mathrm{CH}), 93.4(\mathrm{CH}), 80.0(\mathrm{CH}), 58.4\left(\mathrm{CH}_{3}\right), 55.3$ $\left(\mathrm{CH}_{3}\right), 41.9\left(\mathrm{CH}_{2}\right) ; \mathrm{MS}(\mathrm{ESI}) \mathrm{m} / z 272[\mathrm{M}+\mathrm{Na}]^{+}, 250[\mathrm{M}+\mathrm{H}]^{+}, 121\left[\mathrm{C}_{8} \mathrm{H}_{9} \mathrm{O}\right]^{+}$; HRMS (ESI) calcd for $\mathrm{C}_{13} \mathrm{H}_{15} \mathrm{NO}_{4} \mathrm{Na}[\mathrm{M}+\mathrm{Na}]^{+} 272.0893$, found 272.0883 .

5-Hydroxy-4-methoxy-1-phenyl-1 $H$-pyrrol-2(5H)-one $\quad(6 \mathrm{~g})$. Purification by flash chromatography (hexane/EtOAc 40:60) afforded $\mathbf{6 g}$ as a colourless solid (70\%): mp 138-139 ${ }^{\circ} \mathrm{C}$; IR (thin film) 3271, 1676, $1641 \mathrm{~cm}^{-1}$; ${ }^{1} \mathrm{H}$ NMR $\left(300 \mathrm{MHz}, \mathrm{CDCl}_{3}\right) \delta 7.67$ (dd, $J=8.7,1.0 \mathrm{~Hz}$, 2H), 7.33 (app t, $J=7.5 \mathrm{~Hz}, 2 \mathrm{H}$ ), 7.11 (app t, $J=7.5 \mathrm{~Hz}, 1 \mathrm{H}$ ), 5.67 (br s, 1H), 4.89 (s, 1H), 4.63 (br s, 1H), 3.70 (s, 3H); ${ }^{13} \mathrm{C}$ NMR (75.5 MHz, $\mathrm{CDCl}_{3}$ ) $\delta 173.3(\mathrm{C}), 170.1(\mathrm{C}), 137.3(\mathrm{C}), 128.9$ $(\mathrm{CH}), 124.3(\mathrm{CH}), 120.5(\mathrm{CH}), 93.9(\mathrm{CH}), 81.7(\mathrm{CH}), 58.5\left(\mathrm{CH}_{3}\right)$; MS (ESI) $m / z 228[\mathrm{M}+\mathrm{Na}]^{+}$, $206[\mathrm{M}+\mathrm{H}]^{+}, 188[\mathrm{M}-\mathrm{OH}]^{+}, 174\left[\mathrm{M}-\mathrm{OCH}_{3}\right]^{+}$; HRMS (ESI) calcd for $\mathrm{C}_{11} \mathrm{H}_{12} \mathrm{NO}_{3}[\mathrm{M}+\mathrm{H}]^{+}$ 206.0812, found 206.0811 .

4,5-Dimethoxy-1H-pyrrol-2(5H)-one (7). To a cooled $\left(0{ }^{\circ} \mathrm{C}\right)$ solution of $6 \mathbf{a}(500 \mathrm{mg}, 3.87$ $\mathrm{mmol})$ in $\mathrm{MeOH}(20 \mathrm{~mL})$ was added anhydrous $\mathrm{HCl}$ in $\mathrm{MeOH}(1.0 \mathrm{M}, 775 \mu \mathrm{L}, 0.775 \mathrm{mmol}, 0.20$ equiv.), generated by adding $\mathrm{TMSCl}(256 \mu \mathrm{L}, 2.00 \mathrm{mmol})$ to $\mathrm{MeOH}(2.0 \mathrm{~mL})$. The resulting solution was allowed to stir whilst warming to rt. After $3.5 \mathrm{~h}$, the solvent was removed in vacuo to give 7 (554 mg, 100\%) as a light yellow solid. A small portion of 7 was purified by flash chromatography (EtOAc/ $\mathrm{CH}_{2} \mathrm{Cl}_{2} / \mathrm{MeOH} 50: 50: 1$ to 50:50:5) to afford an analytically pure sample of 7 as a white solid: $\mathrm{mp} 105-106^{\circ} \mathrm{C}$, lit. ${ }^{2} \mathrm{mp} 107^{\circ} \mathrm{C}$; $\mathrm{IR}$ (KBr disc) 3246 (br), 3092, 1701, 1666, $1632 \mathrm{~cm}^{-1} ;{ }^{1} \mathrm{H}$ NMR $\left(400 \mathrm{MHz}, \mathrm{CDCl}_{3}\right) \delta 6.11(\mathrm{br} \mathrm{s}, 1 \mathrm{H}), 5.33(\mathrm{~d}, J=1.9 \mathrm{~Hz}, 1 \mathrm{H}), 5.08(\mathrm{~d}, J=$ $1.3 \mathrm{~Hz}, 1 \mathrm{H}), 3.85$ (s, 3H), 3.29 (s, 3H); ${ }^{13} \mathrm{C}$ NMR (100.6 MHz, $\left.\mathrm{CDCl}_{3}\right) \delta 173.6(\mathrm{C}), 173.4(\mathrm{C})$, $95.0(\mathrm{CH}), 83.9(\mathrm{CH}), 58.7\left(\mathrm{CH}_{3}\right), 52.3\left(\mathrm{CH}_{3}\right)$; MS (+ESI) m/z $144[\mathrm{M}+\mathrm{H}]^{+}, 112\left[\mathrm{M}-\mathrm{OCH}_{3}\right]^{+}$; HRMS (+ESI) calcd for $\mathrm{C}_{6} \mathrm{H}_{10} \mathrm{NO}_{3}[\mathrm{M}+\mathrm{H}]^{+}$144.0655, found 144.0656. Spectroscopic data matched that previously reported. ${ }^{2}$ 
<smiles>COC1=CC(O)N(Cc2ccccc2)C1=O</smiles>

S1

Reduction of 5e with DIBAL-H to give 6e and S1. To a $0{ }^{\circ} \mathrm{C}$ solution of 1-benzyl-3-methoxy1H-pyrrole-2,5-dione (5e) (95.5 mg, $0.440 \mathrm{mmol}, 1.0$ equiv.) in THF (2.2 mL) was added DIBAL-H (1.0 M in heptane, $0.46 \mathrm{~mL}, 0.46 \mathrm{mmol}, 0.462 \mathrm{mmol}, 1.05$ equiv.) dropwise. The resultant brown solution was stirred at $0{ }^{\circ} \mathrm{C}$ for $4 \mathrm{~h}$ after which time t.l.c analysis revealed incomplete consumption of the starting material. The reaction was quenched with half-saturated aq. $\mathrm{NaHCO}_{3}$ solution then diluted with $\mathrm{H}_{2} \mathrm{O}$ and extracted with EtOAc $(3 \times)$. The combined organic extracts were dried $\left(\mathrm{Na}_{2} \mathrm{SO}_{4}\right)$, filtered and concentrated to give a crude brown oil. The products 6e and S1 were separated from the starting material by flash chromatography (EtOAc/acetone 90:10). Separation of a small portion of $\mathbf{S 1}\left(\mathrm{R}_{\mathrm{t}} \sim 12.5 \mathrm{~min}\right)$ and $\mathbf{6 e}\left(\mathrm{R}_{\mathrm{t}} \sim 14.0 \mathrm{~min}\right)$ was achieved by preparative HPLC (EtOAc/i-PrOH 97.5:2.5, $13.5 \mathrm{mLmin}^{-1}$ ), providing S1 as a colourless solid: ${ }^{1} \mathrm{H}$ NMR (300 MHz, $\left.\mathrm{CDCl}_{3}\right) \delta$ 7.34-7.25 (m, 5H), $5.57(\mathrm{~d}, J=2.1 \mathrm{~Hz}, 1 \mathrm{H}), 5.17$ $(\mathrm{d}, J=1.9 \mathrm{~Hz}, 1 \mathrm{H}), 4.94(\mathrm{~d}, J=14.8 \mathrm{~Hz}, 1 \mathrm{H}), 4.27$ (d, $J=14.9 \mathrm{~Hz}, 1 \mathrm{H}), 3.76$ (s, 3H), O프 signal not visible; ${ }^{13} \mathrm{C}$ NMR (75.5 MHz, $\left.\mathrm{CDCl}_{3}\right) \delta 164.2(\mathrm{C}), 153.0(\mathrm{C}), 136.8(\mathrm{C}), 128.7(\mathrm{CH}), 128.4$ $(\mathrm{CH}), 127.6(\mathrm{CH}), 106.8(\mathrm{CH}), 78.9(\mathrm{CH}), 57.4\left(\mathrm{CH}_{3}\right), 42.9\left(\mathrm{CH}_{2}\right)$; MS (ESI) $m / z 242[\mathrm{M}+\mathrm{Na}]^{+}$, $220[\mathrm{M}+\mathrm{H}]^{+}, 202[\mathrm{M}-\mathrm{OH}]^{+}$; HRMS (ESI) calcd for $\mathrm{C}_{12} \mathrm{H}_{13} \mathrm{NO}_{3} \mathrm{Na}[\mathrm{M}+\mathrm{Na}]^{+} 242.0788$, found 242.0787 . 


\section{Single crystal $x$-ray diffraction experimental for compound 6e}

A colourless block-like crystal was attached with Exxon Paratone N, to a short length of fibre supported on a thin piece of copper wire inserted in a copper mounting pin. The crystal was quenched in a cold nitrogen gas stream from a commercial cryosystem. A CCD detector equipped diffractometer employing graphite monochromated $\mathrm{MoK} \alpha$ radiation generated from a rotating anode was used for the data collection. Cell constants were obtained from a least squares refinement against 8777 reflections located between 6 and $56^{\circ} 2 \theta$. Data were collected at 150(2) Kelvin with $\omega$ and $\phi$ scans to $61^{\circ} 2 \theta$. The data integration and reduction were undertaken with SAINT and XPREP, ${ }^{4}$ and subsequent computations were carried out with the WinGX ${ }^{5} \mathrm{XTAL}^{6}$ graphical user interfaces. Absorption was not sufficient to require a correction.

The structure was solved in the space group $P$ na2 $2_{1}(\# 33)$ by direct methods with SIR97, ${ }^{7}$ and extended and refined with SHELXL-97. ${ }^{8}$ The asymmetric unit contains two crystallographically independent molecules. The non-hydrogen atoms were modelled with anisotropic displacement parameters, and a riding atom model with group displacement parameters was used for the hydrogen atoms. Anomalous dispersion effects were not sufficient to determine the absolute structure and accordingly Friedel pairs were merged. An ORTEP ${ }^{9}$ depiction of the molecule with 50\% displacement ellipsoids is provided in Figure S1. 
Figure S1: ORTEP Depiction of $6 \mathbf{e}$, with 50\% displacement ellipsoids.

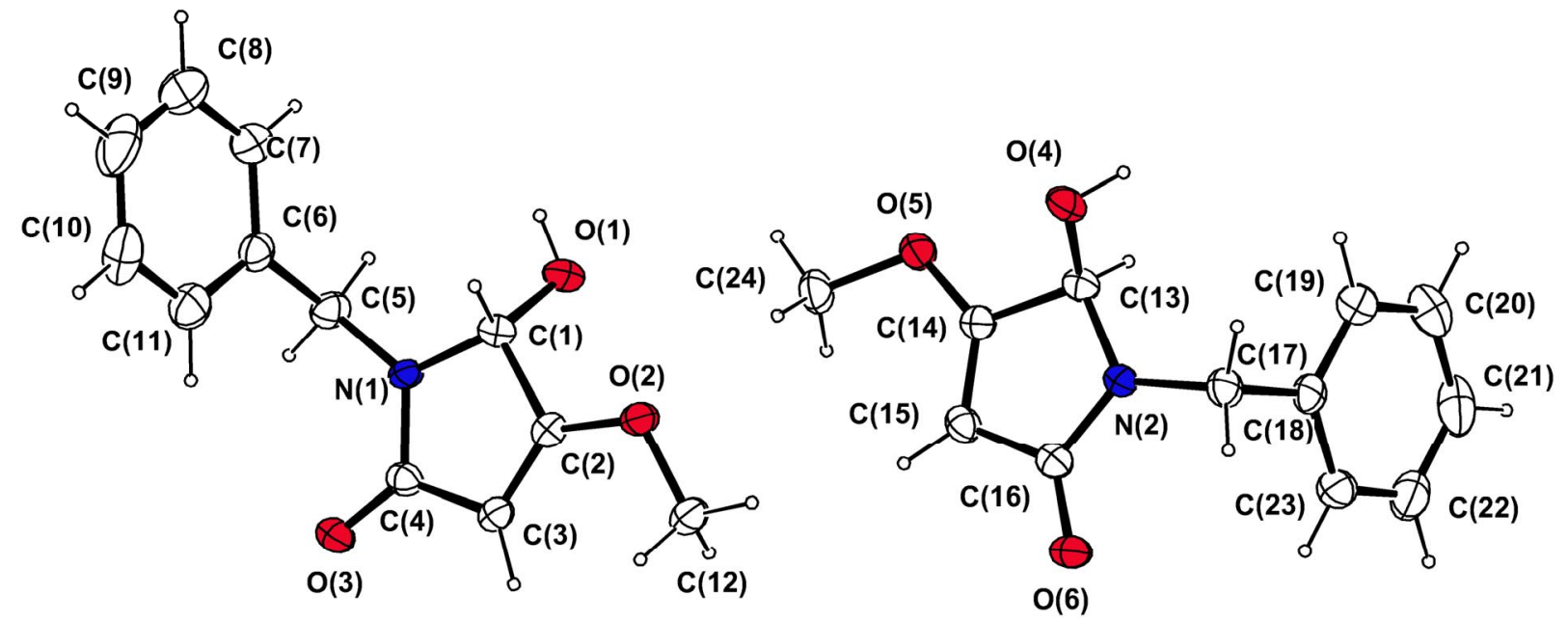

Formula $\mathrm{C}_{12} \mathrm{H}_{13} \mathrm{NO}_{3}, \quad M$ 219.23, Orthorhombic, space group Pna2 ${ }_{1}(\# 33)$, a 8.6992(3), $b$ 6.3159(2), c 39.6797(15) $, V 2180.13(13) \AA^{3}, D_{\mathrm{c}} 1.336 \mathrm{~g} \mathrm{~cm}^{-3}, Z$ 8, crystal size 0.472 by 0.130 by $0.117 \mathrm{~mm}$, colour colourless, habit block, temperature 150(2) Kelvin, $\lambda(\mathrm{MoK} \alpha) 0.71073 \AA$, $\mu(\mathrm{MoK} \alpha) 0.097 \mathrm{~mm}^{-1}, 2 \theta_{\max } 60.92, h k l$ range $-1212,-8$ 8, -52 50, N 30509, $N_{\text {ind }} 5902\left(R_{\text {merge }}\right.$ $0.0946), N_{\text {obs }} 3707(\mathrm{I}>2 \sigma(\mathrm{I})), N_{\text {var }} 293$, residuals ${ }^{*} R 1(F) 0.0365, w R 2\left(F^{2}\right) 0.0689$, GoF(all) 1.184, $\Delta \rho_{\text {min,max }}-0.272,0.168 \mathrm{e}^{-} \AA^{-3}$.

${ }^{*} R 1=\Sigma|| F_{\mathrm{o}}|-| F_{\mathrm{c}}|/ \Sigma| F_{\mathrm{o}} \mid$ for $F_{\mathrm{o}}>2 \sigma\left(F_{\mathrm{o}}\right) ; w R 2=\left(\Sigma \mathrm{w}\left(F_{\mathrm{o}}{ }^{2}-F_{\mathrm{c}}{ }^{2}\right)^{2} / \Sigma\left(\mathrm{w} F_{\mathrm{c}}{ }^{2}\right)^{2}\right)^{1 / 2}$ all reflections $\mathrm{w}=1 /\left[\sigma^{2}\left(F_{\mathrm{o}}{ }^{2}\right)+(0.02 \mathrm{P})^{2}\right]$ where $\mathrm{P}=\left(F_{\mathrm{o}}{ }^{2}+2 F_{\mathrm{c}}{ }^{2}\right) / 3$ 


\section{References}

1. Gill, G. B.; James, G. D.; Oates, K. V.; Pattenden, G. J. Chem. Soc., Perkin Trans. 1 1993, 2567-2579.

2. Farina, F.; Victoria Martin, M.; Carmen Paredes, M.; Carmen Ortega, M.; Tito, A. Heterocycles 1984, 22, 1733-1739.

3. James, G. D.; Mills, S. D.; Pattenden, G. J. Chem. Soc., Perkin Trans. 1 1993, 2581-2584.

4. Bruker. SMART, SAINT and XPREP. Area detector control and data integration and reduction software. Bruker Analytical X-ray Instruments Inc.: Madison, WI, USA, 1995.

5. Farrugia, L. J. J. Appl. Cryst. 1999, 32, 837-838.

6. Hall, S. R.; du Boulay, D. J.; Olthof-Hazekamp, R., Eds., Xtal3.6 System, University of Western Australia: Australia, 1999.

7. Altomare, A.; Burla, M. C.; Camalli, M.; Cascarano, G. L.; Giacovazzo, C.; Guagliardi, A.; Moliterni, A. G. G.; Polidori, G.; Spagna, R. J. Appl. Cryst. 1998, 32, 115-119.

8. Sheldrick, G. M. SHELX97 Programs for Crystal Structure Analysis. University of Göttingen: Germany, 1998.

9. Johnson, C. K. ORTEP II. Report ORNL-5138. Oak Ridge National Laboratory, Oak Ridge, TN, USA, 1976. 


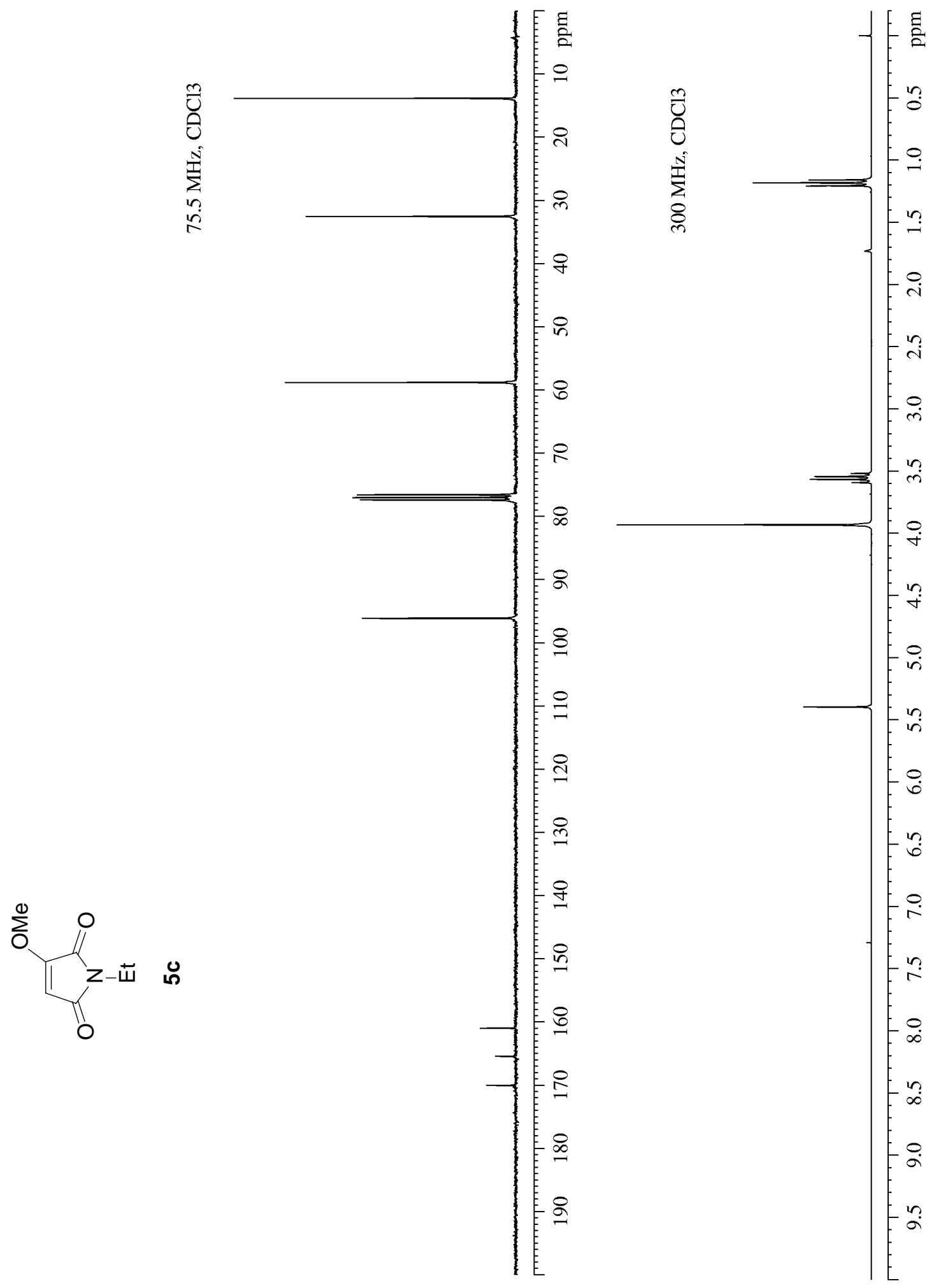




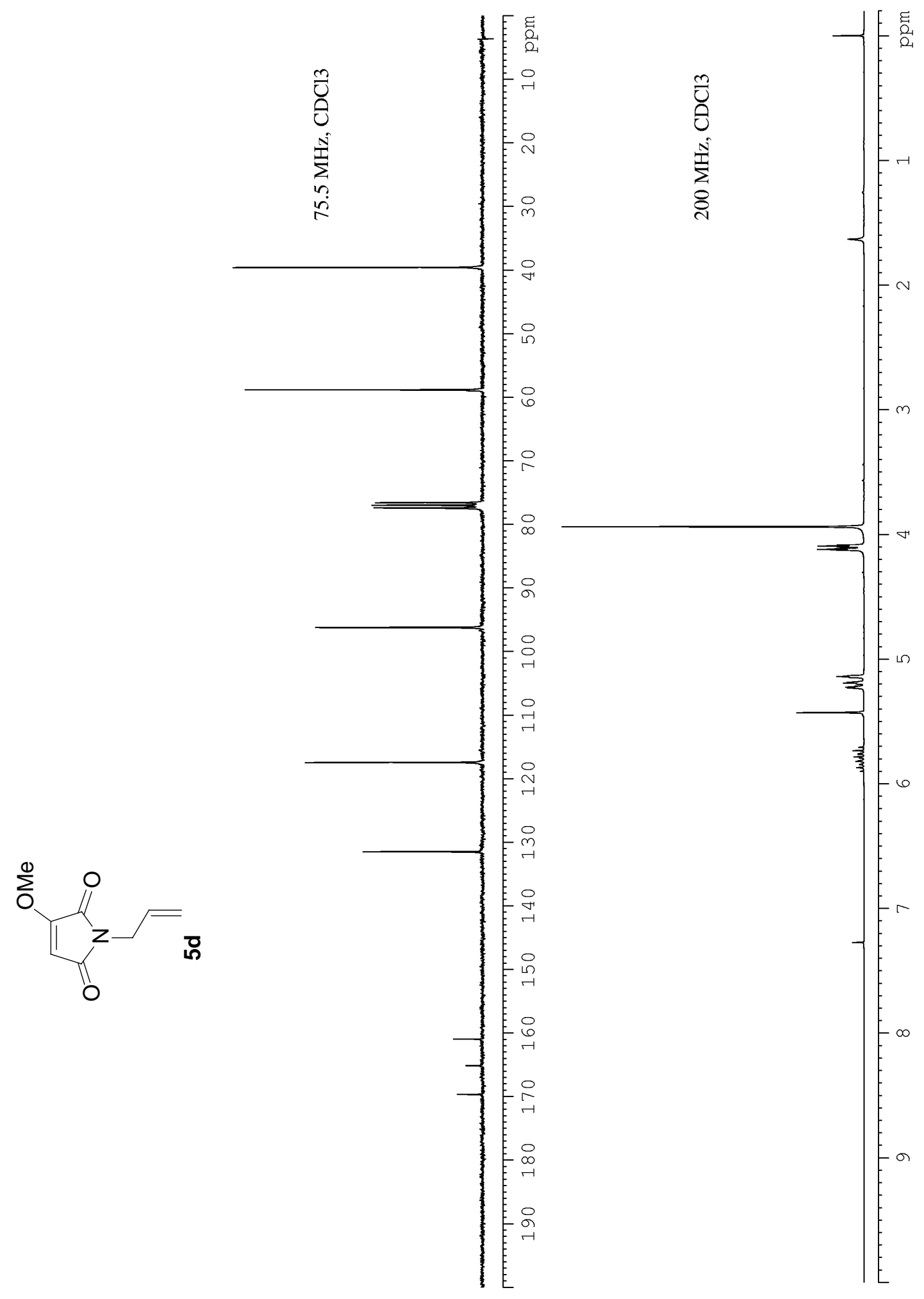




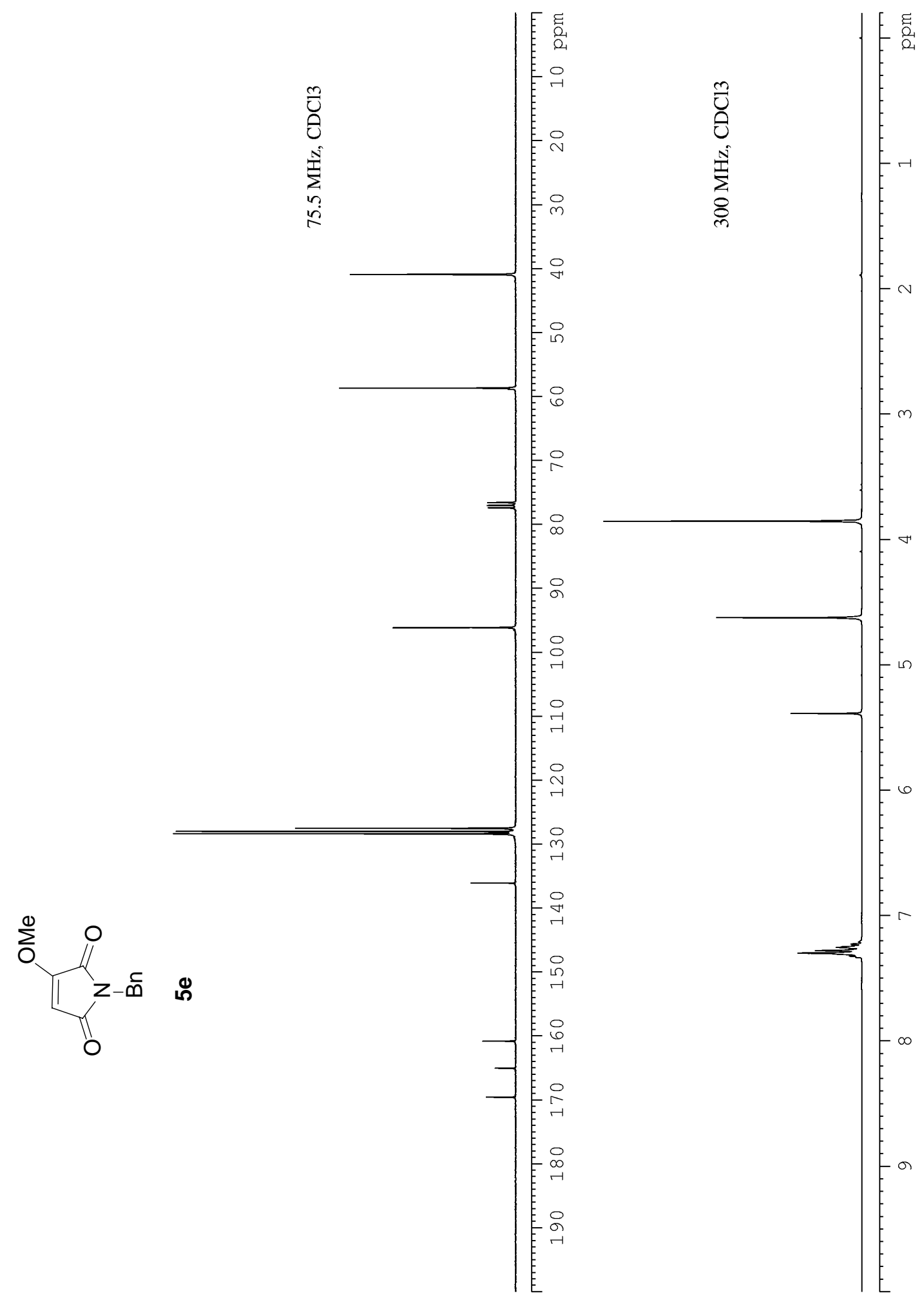




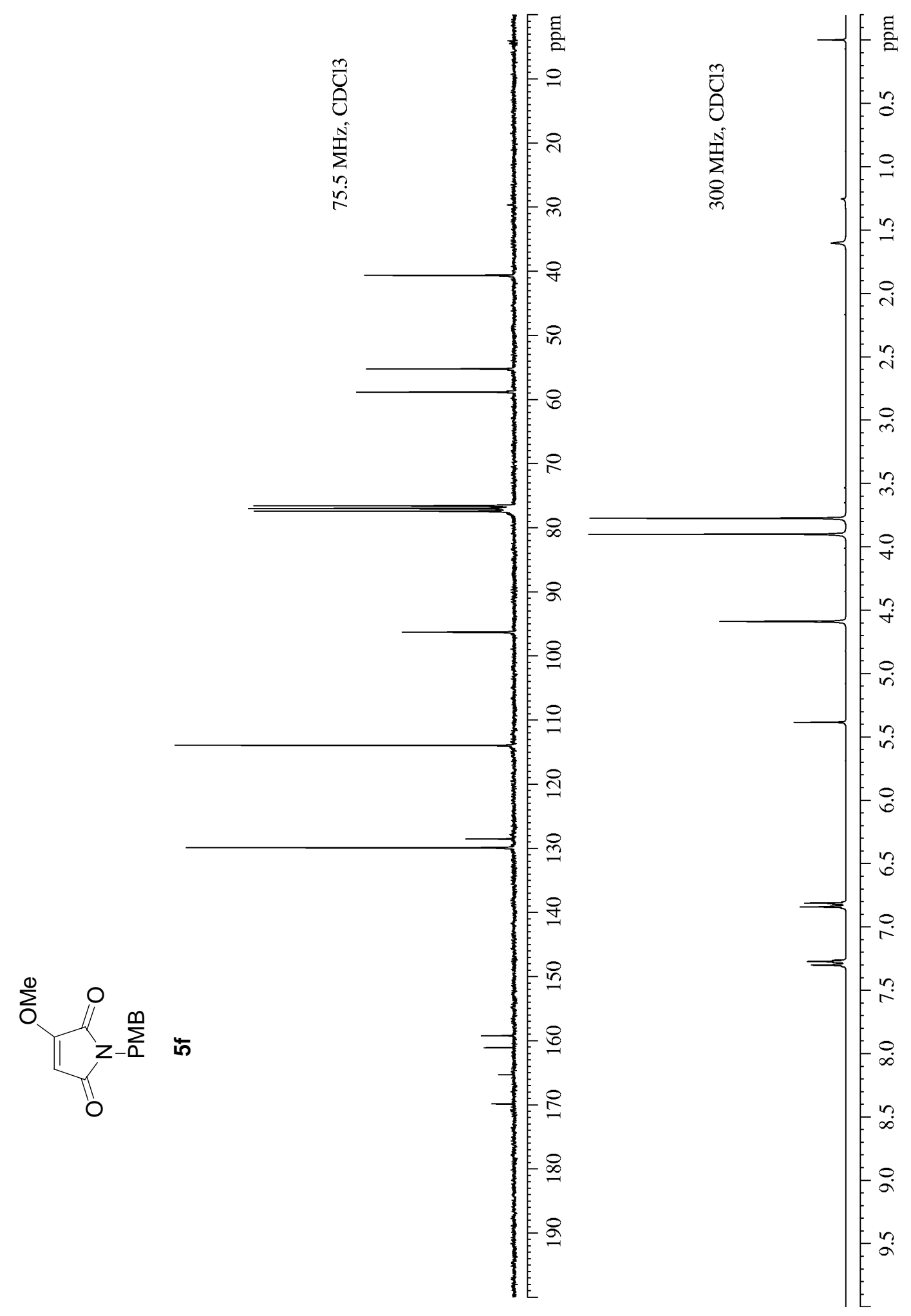




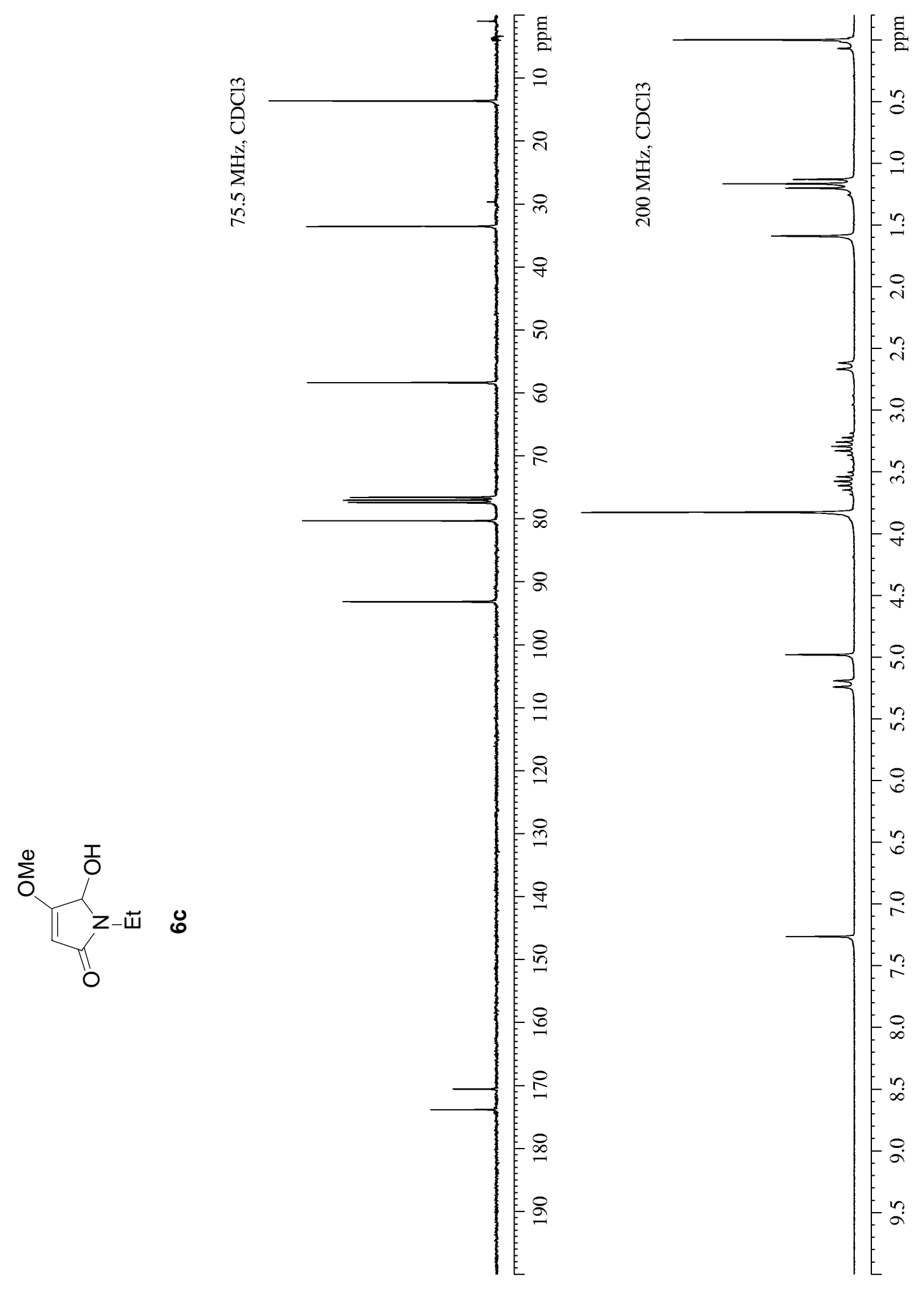




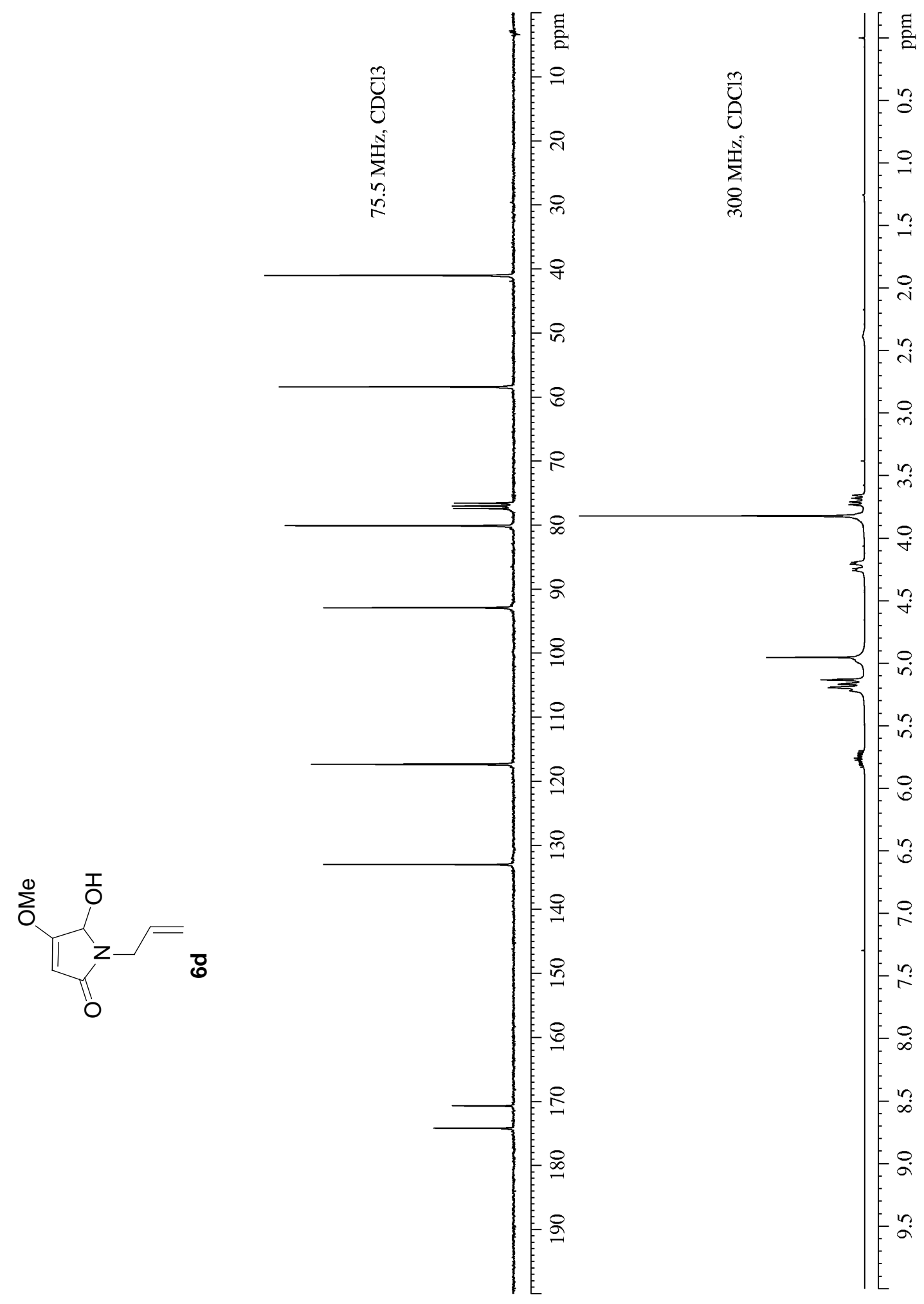




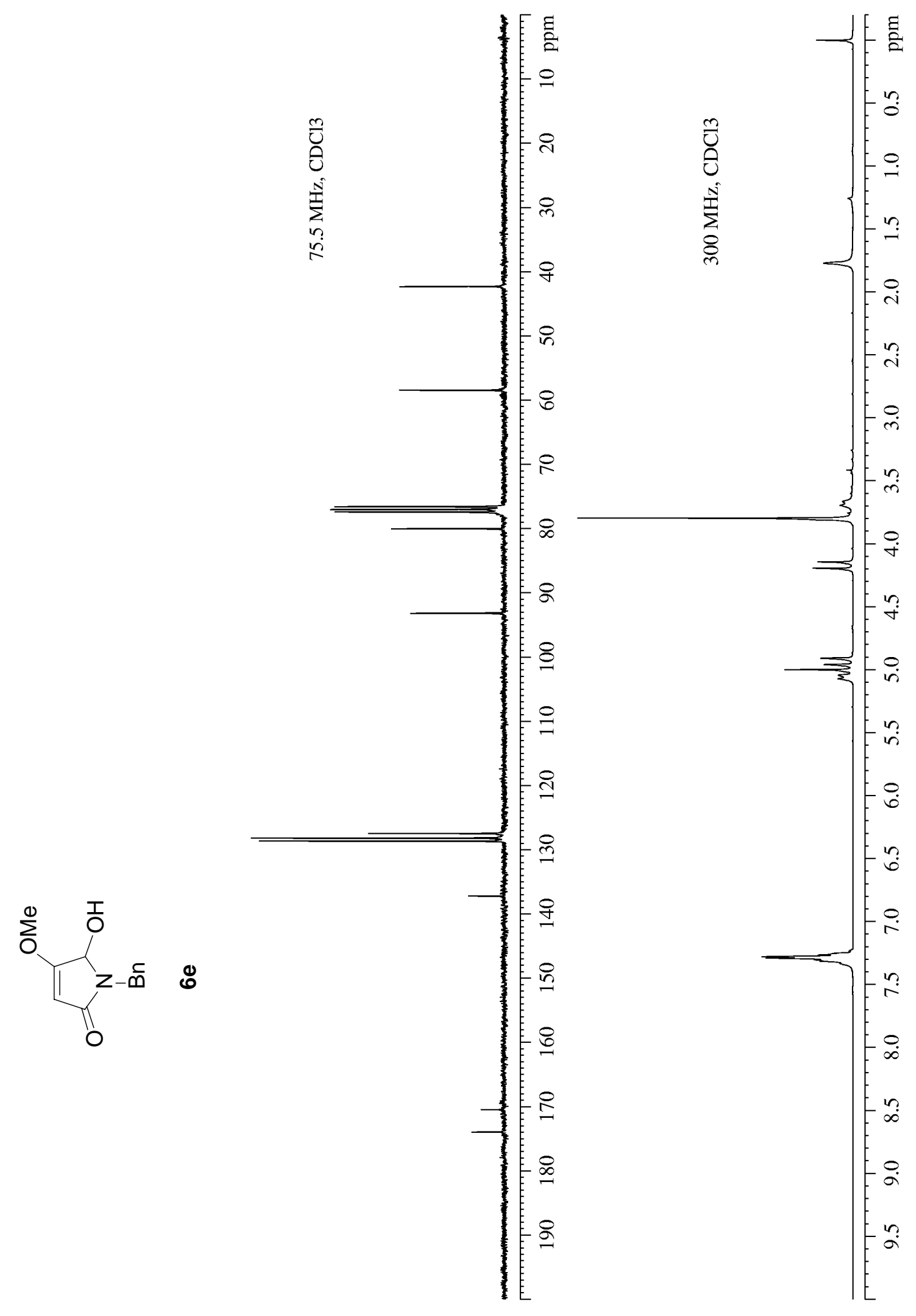




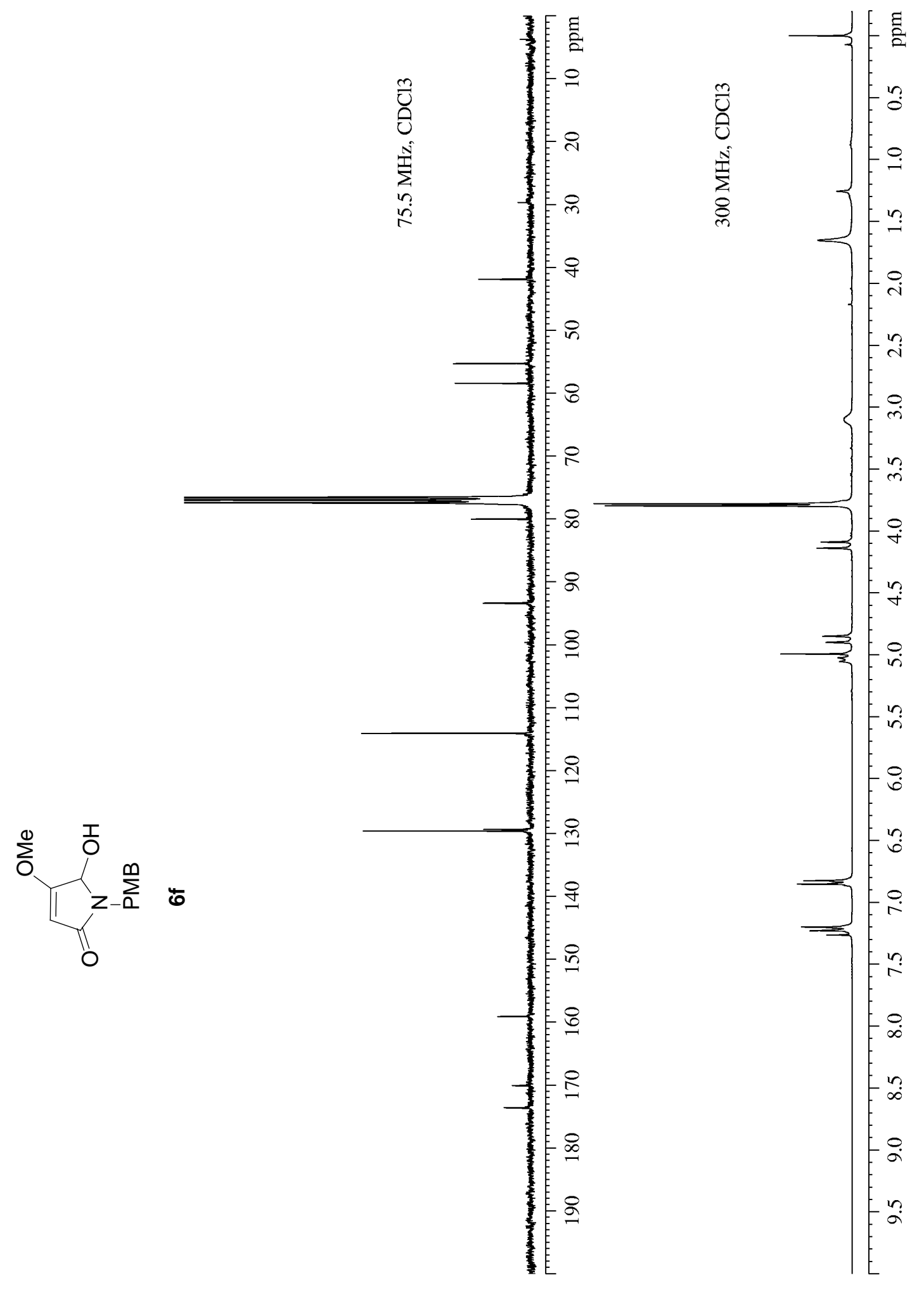




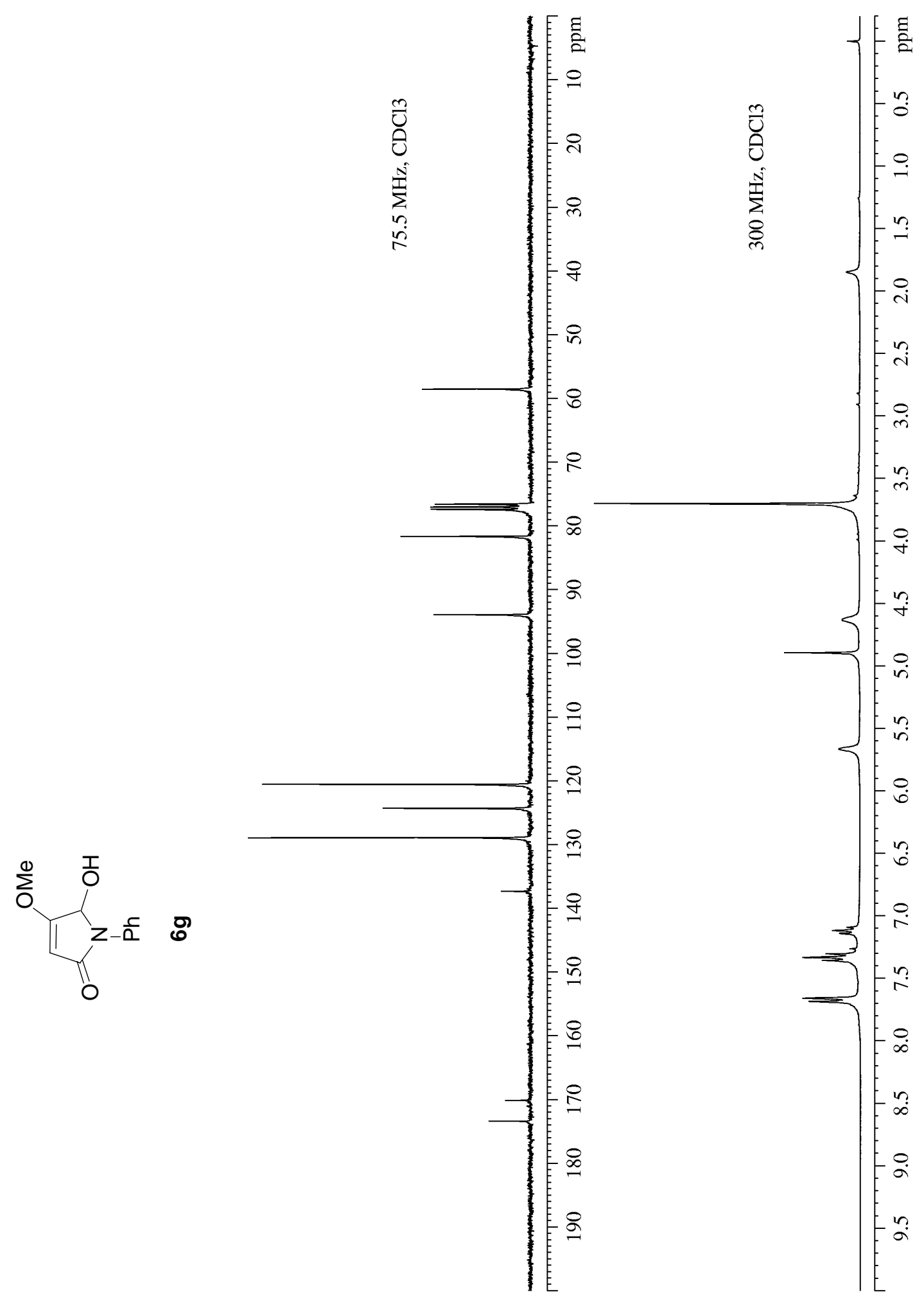




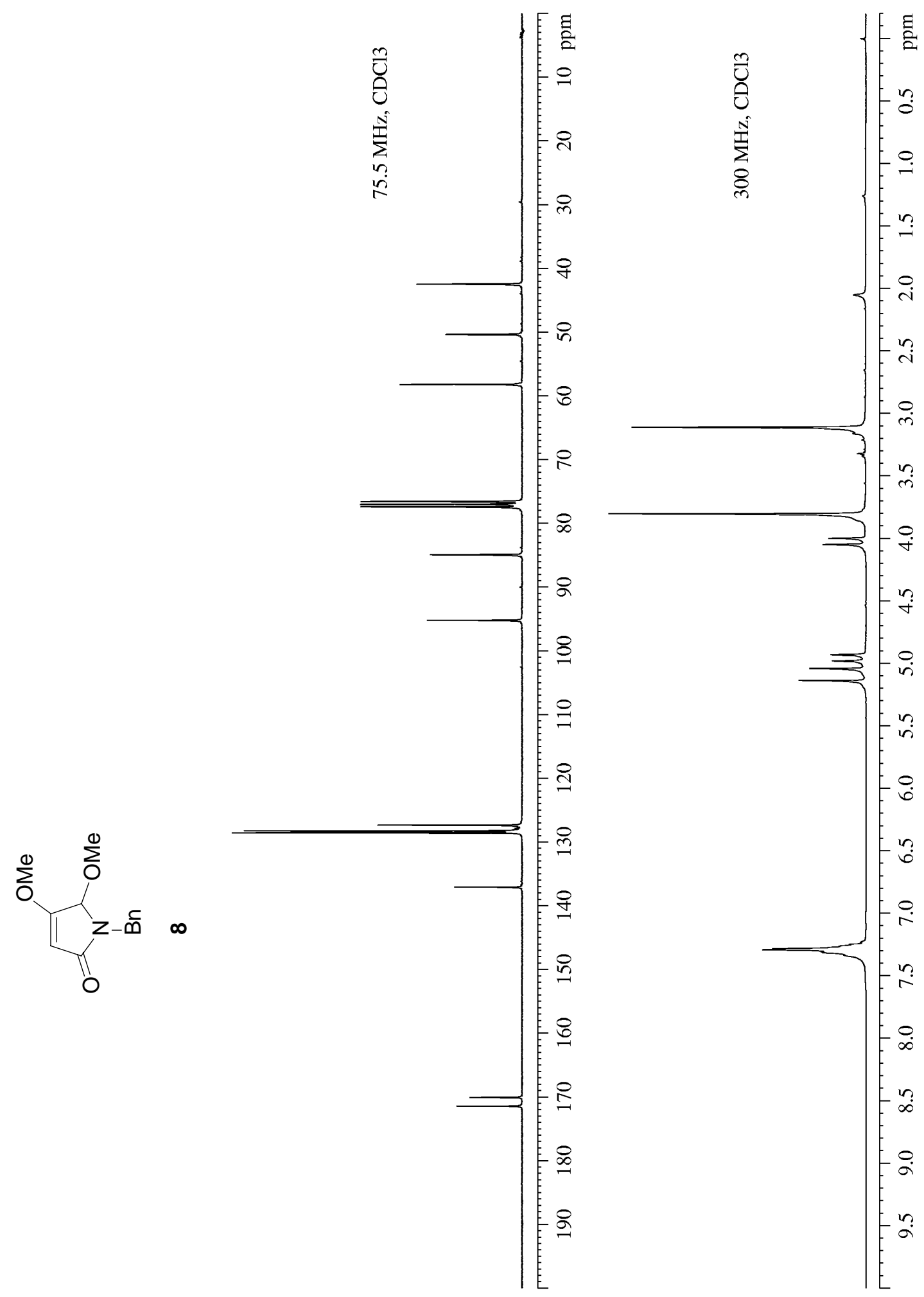




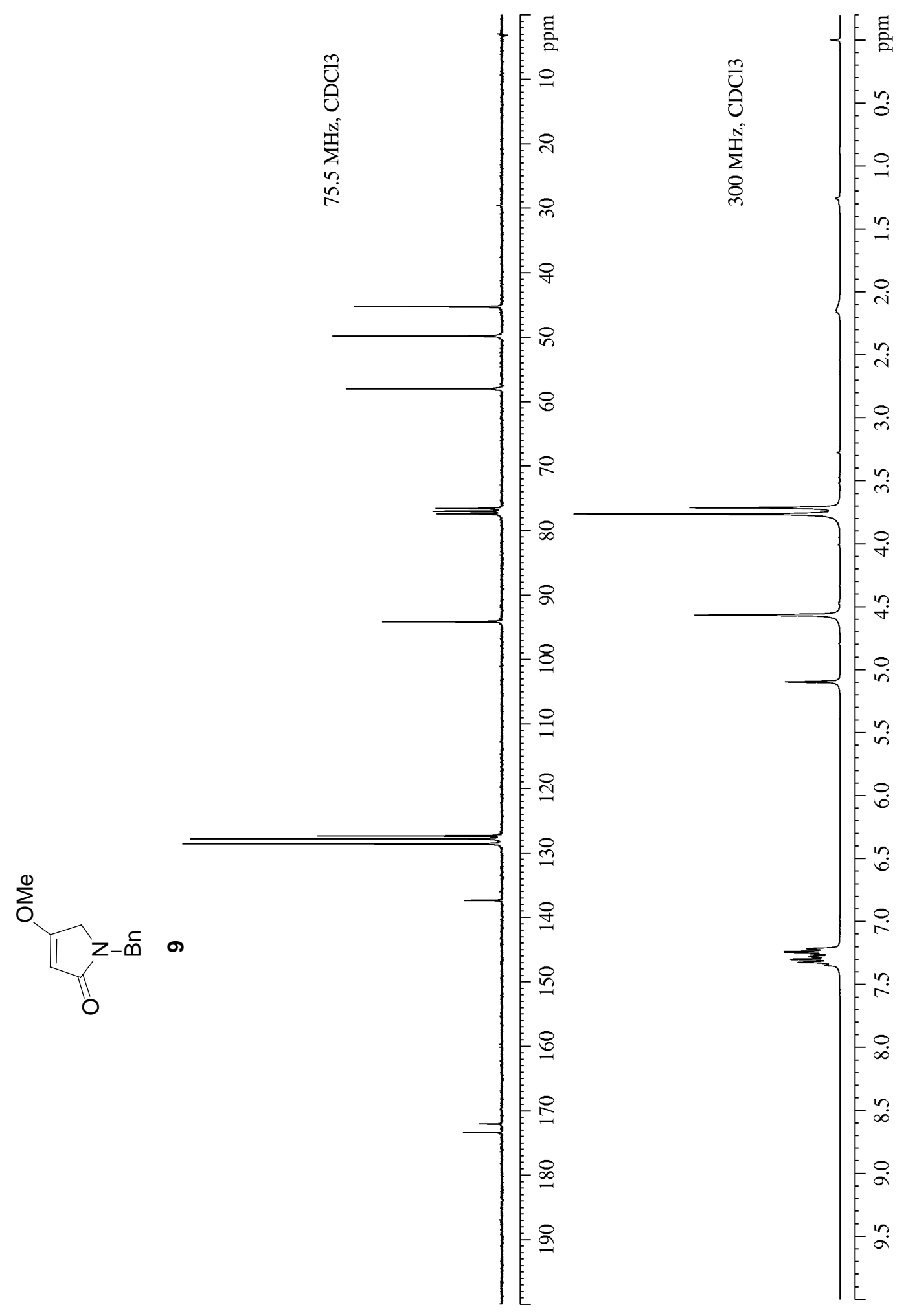




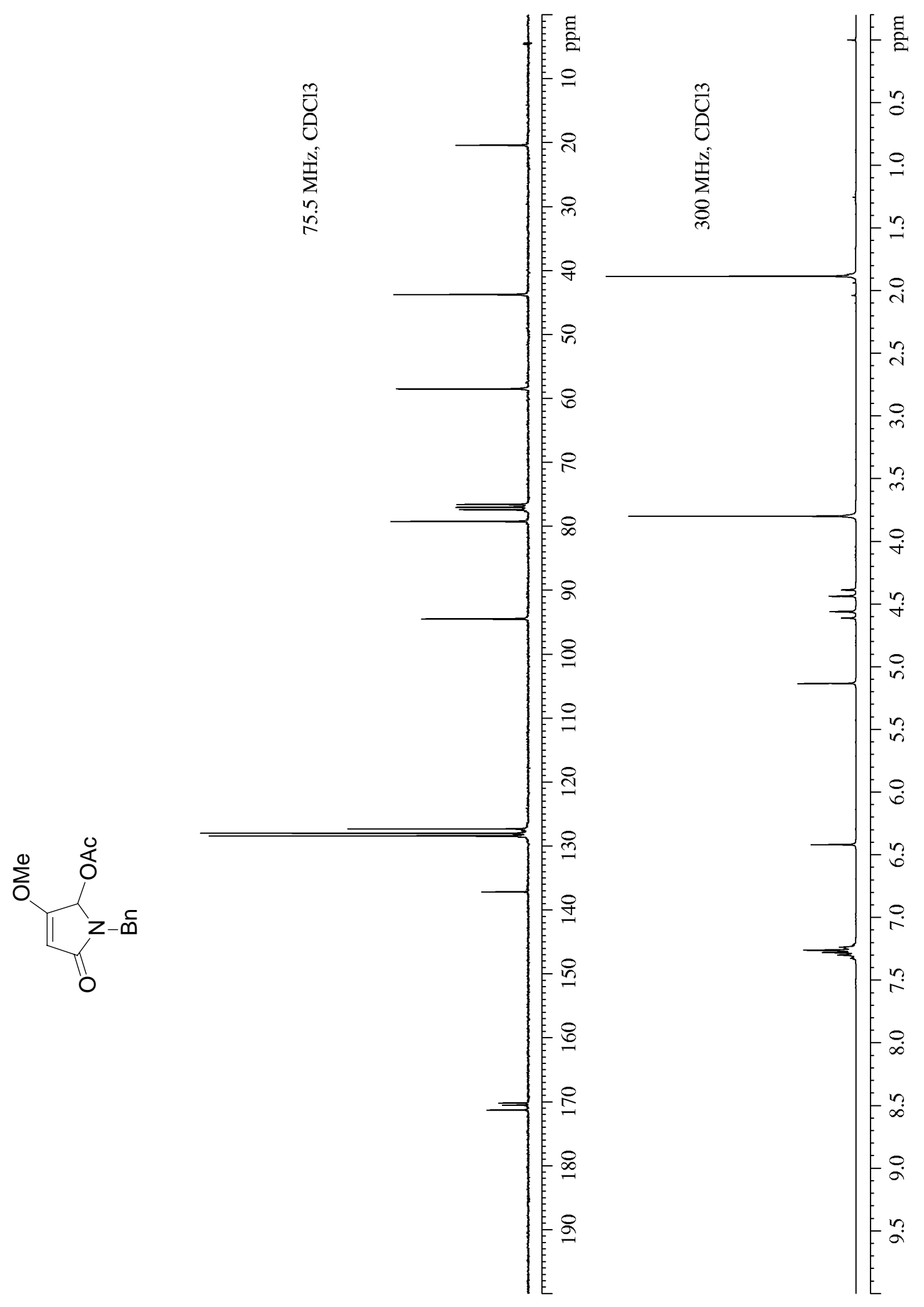




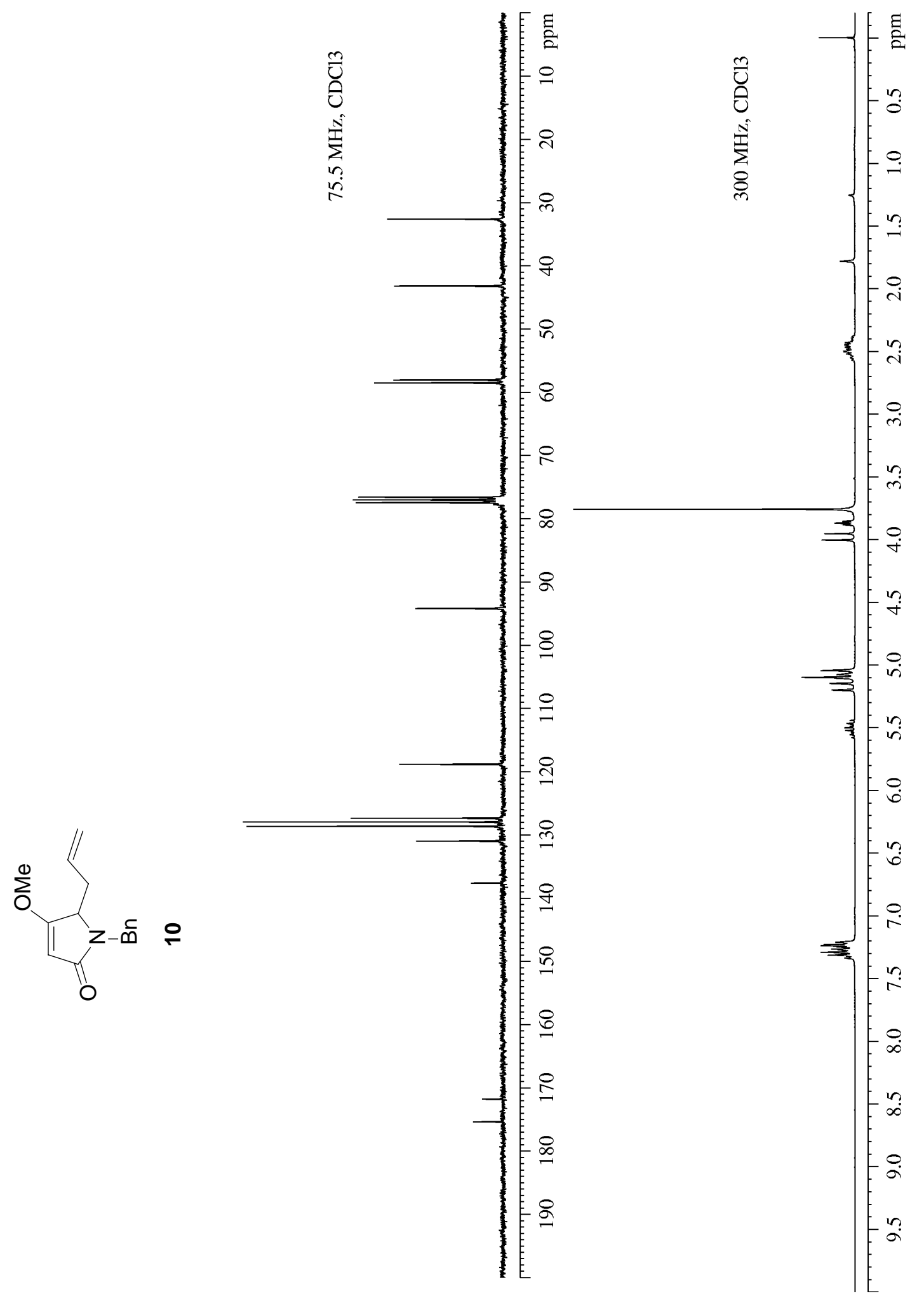




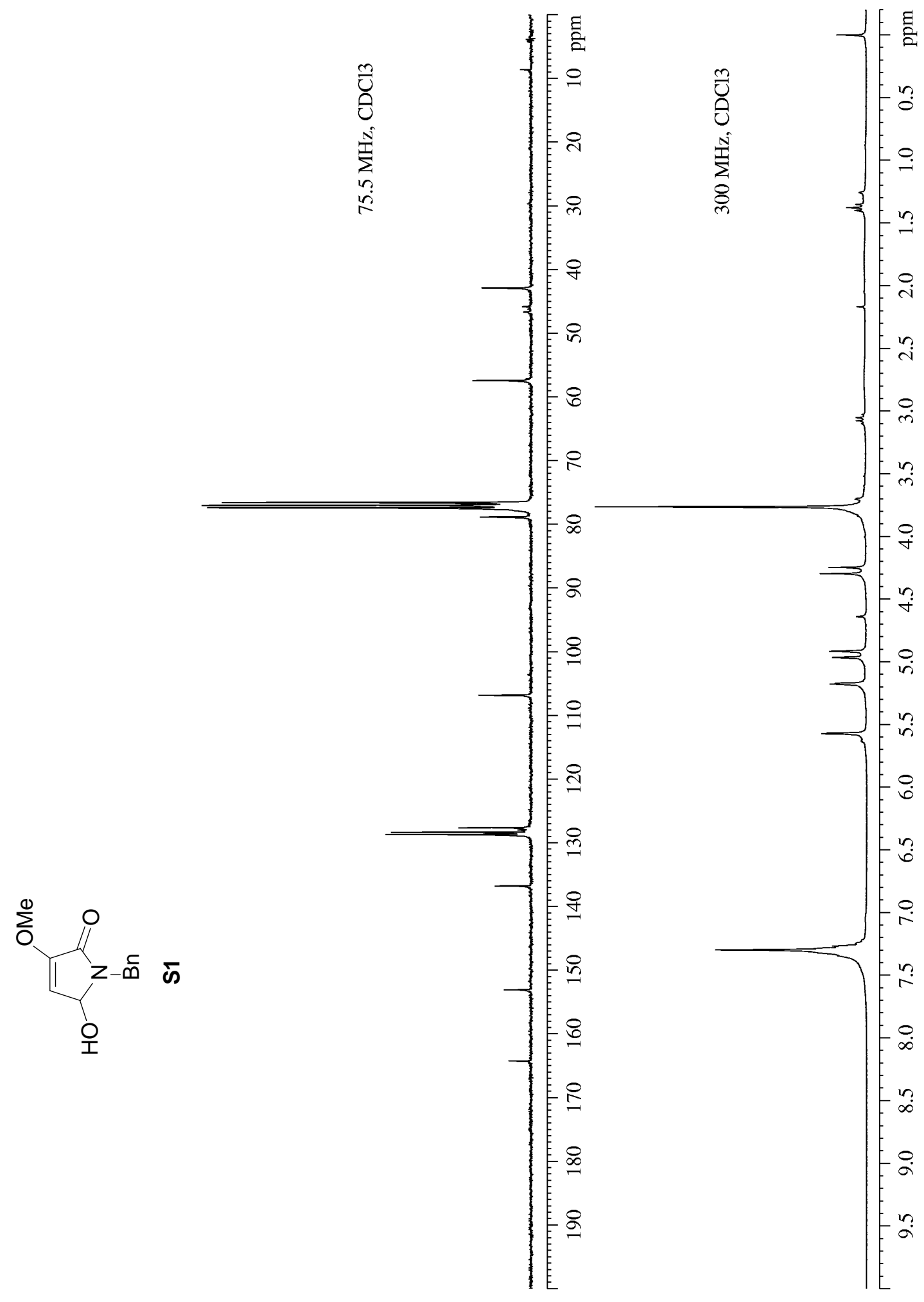

